\title{
Particle and impurity transport in the Axial Symmetric Divertor Experiment Upgrade and the Joint European Torus, experimental observations and theoretical understanding
}

\author{
C. Angioni ${ }^{1}$, L. Carraro ${ }^{2}$, T. Dannert ${ }^{3}$, N. Dubuit ${ }^{4}$, R. Dux ${ }^{1}$, C. Fuchs ${ }^{1}$, X. \\ Garbet $^{4}$, L. Garzotti ${ }^{5}$, C. Giroud ${ }^{5}$, R. Guirlet ${ }^{4}$, F. Jenko ${ }^{1}$, O.J.W.F. Kardaun ${ }^{1}$, L. \\ Lauro-Taroni ${ }^{2}$, P. Mantica ${ }^{6}$, M. Maslov ${ }^{3}$, V. Naulin ${ }^{7}$, R. Neu ${ }^{1}$, A.G. Peeters ${ }^{1}$, G. \\ Pereverzev $^{1}$, M.E. Puiatti ${ }^{2}$, T. Pütterich ${ }^{1}$, J. Stober ${ }^{1}$, M. Valovič ${ }^{5}$, M. Valisa ${ }^{2}$, H. \\ Weisen $^{3}$, A. Zabolotsky ${ }^{3}$, ASDEX Upgrade Team and JET EFDA contributors* \\ 1 Max-Planck Institut für Plasmaphysik, IPP-Euratom Association, \\ D-85748 Garching bei München, Germany \\ ${ }^{2}$ Consorzio RFX, Associazione Euratom-ENEA sulla Fusione, I-35127 Padova, Italy \\ 3 Centre de Recherches en Physique des Plasmas, \\ Association Euratom-Confédération Suisse, \\ EPFL, 1015 Lausanne, Switzerland \\ ${ }^{4}$ CEA-Cadarache, Association Euratom-CEA, 13108 St Paul-les-Durance France \\ ${ }^{5}$ UKAEA-Fusion, United Kingdom Atomic Energy Authority, Abingdon, UK \\ ${ }^{6}$ Istituto di Fisica del Plasma, Associazione Euratom-ENEA-CNR, Milano, Italy \\ ${ }^{7}$ Riso National Laboratory, Association Euratom-Riso, Roskilde, Denmark \\ * See the Appendix of J. Pamela et al., Nucl. Fusion 45, S63 (2005).
}

(Dated: January 22, 2007)

\begin{abstract}
Experimental observations on core particle and impurity transport from the Axial Symmetric Divertor Experiment Upgrade [O.Gruber, H.-S. Bosch, S. Günter et al., Nucl Fusion 39, 1321 (1999)] and the Joint European Torus [J. Pamela, E.R. Solano, and JET EFDA Contributors, Nucl. Fusion 43, 1540 (2003)] tokamaks are reviewed and compared. Robust general experimental behaviours observed in both the devices and related parametric dependences are identified. The experimental observations are compared with the most recent theoretical results in the field of core particle transport.
\end{abstract}




\section{INTRODUCTION AND MOTIVATIONS}

Recently, a large part of the research on core transport in the Axial Symmetric Divertor Experiment (ASDEX) Upgrade (AUG) [1] and the Joint European Torus (JET) [2] tokamaks has been dedicated to particle [3-15]. and impurity transport [16-23].

Particle transport, namely the transport of electrons, main ions and impurities, is an essential ingredient in the physics understanding of transport in tokamak plasmas. Heat and particle transport channels are often strongly coupled, and the general physics understanding of core transport cannot be obtained by focusing exclusively on the heat transport channels. Moreover, the experimental observations on both heat and particle transport considered together provide much more stringent validation tests for theoretical models than observations on heat transport alone.

Particle transport determines the shape of the electron, main ions and impurity profiles. This has important consequences related to the capability of extrapolating present tokamak scenarios to next step devices like the International Thermonuclear Experimental Reactor (ITER) [24], since the shape of the density profile, as well as the existence and magnitude of an inward particle pinch, have several important implications, like the need and amount of fuelling, the influence of the density limit or of the density gradient on magnetohydrodynamical limits, as well as the amount of bootstrap current produced by the plasma. Moreover, a peaked deuterium density profile is prone to produce central impurity accumulation by neoclassical transport [25]. While a peaked deuterium density profile has positive consequences on the fusion performance, highly charged impurity accumulation is disadvantageous since it dilutes the main ions and can cause severe losses by radiation. Therefore, an important step is the identification of plasma conditions which lead to impurity accumulation, in order to be able to predict whether accumulation could take place also in a burning plasma. In combination, external tools to suppress impurity accumulation have to be identified and the physical mechanisms on which they rely have to be assessed in order to verify whether they are likely to be effective in a burning plasma.

While experimentally studies of electron transport and of impurity transport are often performed separately since they involve different diagnostics, it is our opinion that it is useful to consider the problem of particle transport (electron, main ions and impurities) as a single topic of research. This is because the behaviour of the different species is strongly 
coupled in the plasma, and because from the theoretical point of view electrons and ions are governed by the same basic equations, although with different charges and masses. It must be underlined that experimental phenomena of impurity transport can shed light on the behaviour of the electrons as well, when the physics understanding of the impurity transport behaviour is obtained in the framework of a theoretical model.

Finally, the comparison and combination of results from different devices often allows a more complete assessment of the parameter domains within which specific experimental behaviours are observed and a more robust identification of parameter dependences.

To this purpose, the present paper reviews and compares recent experimental results on particle and impurity core transport in AUG and JET, aiming at identifying a set of basic particle and impurity transport behaviours present in both the devices. These experimental behaviours are compared with the most recent theoretical results describing transport caused by microinstabilities present in the plasma core.

In the next section, observations from AUG and JET H-mode plasmas are combined in a single database and the statistically most relevant parameters describing density peaking and empirical scalings with related ITER projections are presented. These empirical results are compared with gyrofluid and gyrokinetic results in Sec. III. In Sec. IV, examples of suppression of impurity accumulation with external heating in AUG and JET are presented, and common properties in the anomalous transport of impurities are discussed. Finally Sec. V compares the experimental behaviour with calculations of impurity transport caused by core microinstabilities.

\section{ELECTRON DENSITY PEAKING IN AUG AND JET H-MODE PLASMAS}

A database of 343 AUG observations and 277 JET observations has been built, starting from previous separate works in the two devices $[4,9,12]$. A set of plasma parameters has been defined in a consistent way and data have been taken from the same kind of diagnostics in the two devices when possible, in such a way to minimize as much as possible systematic errors. In particular a consistent set of density profiles for the two devices has been obtained by computing line integrals of measured JET density profiles with the geometry of the AUG interferometer and then by inverting both computed JET and measured AUG interferometry signals with the same method of projection on a set of basis functions. We 
have adopted the definition of density peaking $n_{e}\left(\rho_{\psi}=0.2\right) /\left\langle n_{e}\right\rangle$ because, among many others, by this definition, the value of density peaking turns out to be strongly constrained by the interferometer line integrals and does not change significantly when changing the set of basis functions (the symbol \langle\rangle denotes a volume average). A multivariate statistical analysis, including regressions in both linear and logarithmic forms, and over a large set of plasma parameters, including $\rho_{*}, \nu_{e f f}, \beta, F_{G r}, q_{95}, \delta, T_{e}\left(\rho_{\psi}=0.2\right) /\left\langle T_{e}\right\rangle, R_{g e o}$ and $\Gamma_{N B I}^{*}$, is performed. The following definitions are adopted

$$
\rho_{*}=4.3710^{-3}\left(m_{\mathrm{eff}}\left\langle T_{e}\right\rangle\right)^{0.5} / B_{T} / a, \quad \nu_{\mathrm{eff}}=0.2\left\langle n_{e}\right\rangle R_{g e o} /\left\langle T_{e}\right\rangle^{2}, \quad \beta=4.0210^{-3}\langle p\rangle / B_{T}^{2}
$$

where densities are in $10^{19} \mathrm{~m}^{-3}$, temperatures in $\mathrm{keV}$, magnetic fields in Tesla, the total plasma pressure $p$ in $\mathrm{keV} \times 10^{19} \mathrm{~m}^{-3}$. The Greenwald fraction $F_{G r}=n / N_{G r}$ is included in order to check its relevance in the regression with respect to collisionality, to which it is strongly correlated in the separate databases of the two devices and remains correlated also in the combined database. Geometry parameters like elongation and aspect ratio have been excluded since their variation is limited in AUG and JET. Instead the dimensional parameter $R_{\text {geo }}$ has been introduced as a device label. This allows us to check whether its inclusion in the regressions affects the relevance of some dimensionless variables, or if its relevance in regressions increases when excluding some dimensionless variables. Finally the neutral beam injection (NBI) particle source parameter is defined

$$
\Gamma_{N B I}^{*} \doteq \frac{R \Gamma_{N B I}}{n D}=2 T \frac{\Gamma_{N B I}}{Q_{N B I}} \frac{Q_{N B I}}{Q_{T O T}}\left|\frac{R}{T} \frac{d T}{d r}\right| \frac{\chi}{D}
$$

and quantifies the beam contribution to density peaking. The neutral beam heating and particle source profiles are computed for all the observations in the database by the steadystate Fokker-Planck PENCIL code [26] for JET data and the Monte Carlo FAFNER code [27] for AUG data. The normalized logarithmic temperature gradient $R / T(d T / d r)$ has been expressed in terms of the electron temperature peaking factor $T_{e 2} /\left\langle T_{e}\right\rangle$. A linear regression over a set of well diagnosed AUG and JET profiles reveals that the normalized logarithmic temperature gradient can be expressed by $\left|\frac{R}{T} \frac{d T}{d r}\right|=3.23\left(T_{2} /\langle T\rangle-0.37\right)$ with RMSE normalized to the mean value of $9.72 \%$. Therefore, in the statistical analysis over the full AUG and JET dataset, and consistently with the definition used for the density peaking, we have replaced the logarithmic temperature gradient in Eq. (2) with the quantity $\left(T_{2} /\langle T\rangle-0.37\right)$. Assuming that the ratio $\chi / D$ is a weak function of the plasma parameters, 
which is the strongest assumption in this procedure, it can be treated as a constant. Then all the other terms in the right hand side of Eq. (2) can be evaluated using the parameters available in the database. We note that the definition of $\Gamma_{N B I}^{*}$ in Eq. (2) is such that in a linear regression for the normalized logarithmic density gradient $R / L_{n}$, the regression coefficient of $\Gamma_{N B I}^{*}$ provides an empirical estimate of the average value of $\chi / D$ over the full set of observations of the database. In our approach, in which the regressed variable is the density peaking $n_{e 2} /\left\langle n_{e}\right\rangle$, the regression coefficient of $\Gamma_{N B I}^{*}$ can still be interpreted as the average value of $\chi / D$ provided that it is renormalized to the appropriate factors relating $T_{e 2} /\left\langle T_{e}\right\rangle$ and $n_{e 2} /\left\langle n_{e}\right\rangle$ to the corresponding logarithmic gradients $R / L_{T e}$ and $R / L_{n}$ at mid-radius.

We have performed both linear and logarithmic regressions, although linear regressions are deemed more appropriate given the physical meaning and the range of variation of the regressed variable. According to [28], we define the following parameter to describe the statistical relevance $S t R_{j}$ of the parameter $X_{j}$ in the linear regression $c+\sum_{j} \hat{a}_{j} X_{j}$ for the response variable $Y, S t R_{j}=\hat{a}_{j} \times \operatorname{STD}\left(X_{j}\right)$, where with $\operatorname{STD}$ we denote the usual standard deviation (the same definition is adopted in logarithmic regressions). The parameter $S t R_{j}$ estimates the variation of the regressed variable for one standard deviation variation of the regression variable $X_{j}$, keeping fixed all the other regression variables. Furthermore, we have considered the statistical significance, defined as $S t S_{j}=\hat{a}_{j} / \operatorname{STD}\left(a_{j}\right)$, where $\operatorname{STD}\left(a_{j}\right)$ is one standard deviation, namely a $66.67 \%$ confidence interval, of the estimated regression coefficient $\hat{a}_{j}$. Very similar results have been found with linear and logarithmic regressions. Table 1 shows the statistical relevance of the regression variables in a set of different linear regressions in which $\nu_{e f f}, F_{G r}$ and $R_{g e o}$ are alternatively included and excluded. Plots of the density peaking as a function of a set of physically relevant parameters, namely $\nu_{e f f}, F_{G r}, \rho_{*}$ and $\Gamma_{N B I}^{*}$ are presented in Fig. 1. A more detailed description of the statistical analysis will be reported in a separate publication [14]. The main conclusions of the statistical analysis are the following.

- Collisionality is highly significant, and is the most relevant parameter in all the regression models in which it is included, as shown in Table 1.

- The Greenwald fraction is relevant and significant only if collisionality is excluded.

- Parameters like $\rho_{*}$ and/or $R_{g e o}$ become more relevant when collisionality is excluded. 
In particular $\rho_{*}$ is negligible in regressions with collisionality, whereas at fixed Greenwald fraction, the density peaking increases with increasing size of the device, or with decreasing $\rho_{*}$.

- The beam fuelling parameter is always significant and relevant, as it is shown in Table 1 and as it can be also inferred from the systematic difference between points with ICRH only and points with NBI in Fig. 1 (a) and (b). It can become the most relevant parameter when collisionality is excluded (3rd line in Table 1). However in no regression the beam fuelling is found to be able to describe alone the full observed variation of density peaking. In particular, in regressions with collisionality, it cannot explain more than $30 \%$ of the observed variation of density peaking. Finally, in regressions which include collisionality in the regression variables, the ratio $\chi / D$ is found to be around 1.5, in agreement with previous estimates obtained on the set of JET data alone [12], whereas it is found to be larger (around 2.5) in regressions which include the Greenwald fraction and exclude collisionality.

These points, in addition to the observation of peaked density profiles at low collisionality with radiofrequency heating only, lead to the conclusion that an inward anomalous pinch exists in low collisionality $\mathrm{H}$-mode plasmas, and it is the main cause of the observed peaking.

We mention that a collisionality dependence of density peaking very similar to that observed in AUG and JET has been observed recently also in Alcator C-Mod [29], confirming the existence of an anomalous inward pinch producing peaked density profiles in low collisionalilty $\mathrm{H}$-mode plasmas.

The effects of this anomalous pinch on the ITER electron density profile can be empirically predicted by a regression which includes collisionality together with other significant parameters, like

$$
\begin{aligned}
n_{e 2} /\left\langle n_{e}\right\rangle_{V o l}= & 1.347 \pm 0.014-(0.117 \pm 0.005) \log \left(\nu_{\mathrm{eff}}\right)+ \\
& +(1.331 \pm 0.117) \Gamma_{N B I}^{*}-(4.030 \pm 0.810) \beta
\end{aligned}
$$

with RMSE $=0.115$ (66.7\% confidence intervals for the regression coefficients, corresponding to one standard deviation, are quoted). This scaling predicts a density peaking for ITER $n_{e}\left(\rho_{\psi}=0.2\right) /\left\langle n_{e}\right\rangle \simeq 1.46 \pm 0.04$. With the definition of $\nu_{e f f}$ in Eq. (1), ITER collisionality is 0.19 , namely the ITER prediction can be considered as the value of density peaking in 
the limit of low collisionality and in the absence of beam source $\left(\Gamma_{N B I}^{*}=0\right)$, at zero particle flux. It is equivalent to a dimensionless logarithmic density gradient at mid-radius $R / L_{n}$ between 2.5 and 3.5. These numbers will be compared with the theoretical results in the next section.

The fact that, unlikely in L-mode plasmas [9], no relevance is found for parameters like the safety factor $q_{95}$ or the internal inductance $l_{i}$ is surprising and certainly puzzling. Moreover, in contrast to $\mathrm{H}$-mode plasmas, density profiles of JET L-mode plasmas are observed to depend mainly on the peaking of the current profile and not on the collisionality [9], On the contrary, a collisionality dependence of the peaking of the density profiles has been observed in AUG L-mode plasmas [10]. Finally we mention that on a subset of 114 JET observations in which measurements of both the ion and the electron temperature are available, a positive weak correlation between density peaking and the ion to electron temperature ratio $T_{i} / T_{e}$ is found [12].

\section{COMPARISON WITH THEORETICAL RESULTS}

The theoretically predicted local slope of the density profile at zero particle flux can be computed with present gyrokinetic codes. Usually, these use as input the gradients of the profiles to compute the corresponding fluxes. Therefore, a set of runs at different values of the logarithmic density gradient $R / L_{n}$ allows the identification of the value of $R / L_{n}$ at which the particle flux crosses zero. Of course, in general the particle flux does not depend exclusively on the density gradient, therefore this calculation provides the particle flux dependence on the density gradient for a given set of the other parameters.

For typical parameters at mid-radius of AUG and JET H-mode plasmas, namely $T_{e}=$ $T_{i}, R / L_{T e}=R / L_{T i} \simeq 6$, it is found that, in the collisionless limit, for small values of $R / L_{n}$ the total particle flux is directed inwards, while for large values of $R / L_{n}$ is directed outwards. In Fig. 2a, the behaviour of the particle flux as a function of the logarithmic density gradient $R / L_{n}$ is plotted for different values of the logarithmic temperature gradient $R / L_{T e}=R / L_{T i}=R / L_{T}$ in quasi-linear calculations with the linear version of the GS2 code [30, 31]. For comparison, nonlinear results in the collisionless limit with the GENE code [32] at $R / L_{T}=9$ are also plotted, showing good agreement with the quasi-linear results (other parameters are $\epsilon=0.16$, magnetic shear $s=0.8$ and $q=1.4, T_{e}=T_{i}$ ). The 
details of the quasi-linear model adopted in these calculations are provided in [32, 33]. The intersections at zero particle flux obtained by these calculations are reported in Fig. $2 \mathrm{~b}$. The curve of $R / L_{n}$ at $\Gamma=0$ as a function of $R / L_{T}$ identifies domains of instability for the ion temperature gradient (ITG) and the trapped electron mode (TEM) microinstabilities. At small values of $R / L_{n}$ and $R / L_{T}$ the system is stable, at large values of $R / L_{T}$ and small values of $R / L_{n}$ the instability is an ITG, and the particle flux is directed inwards. Finally, for large values of $R / L_{n}$, the instability is a density gradient driven TEM (e.g. [33-35] ) and the particle flux is directed outwards. Such an instability occurs also at values of $R / L_{T}$ which are below the ITG threshold. In that case, the $R / L_{n}$ driven TEM has a threshold in $R / L_{n}$ (e.g. curve at $R / L_{T}=0$ in Fig. 2a). Since we are mainly presenting results from linear calculations, we have to mention that this threshold can have a nonlinear upshift [34] similar to the one found for the ITG threshold for $R / L_{T i}$ [36]. In Fig. $2 \mathrm{~b}$, the domain of logarithmic temperature gradients usually measured in $\mathrm{H}$-mode plasmas in AUG and JET is also highlighted, $R / L_{T}$ between 4 and 7.5, which is above, but rather close to the ITG threshold. The curve $\Gamma=0$ identifies a corresponding range of values for $R / L_{n}$, which are those predicted in the collisionless limit for experimental values of $R / L_{T}$. These are found to be between 3 and 4, namely in the same range measured in the experiments at low collisionality, and not far from the ITER prediction obtained with the combined AUG-JET scaling for density peaking. We recall that the ITER prediction provided by the scaling corresponds in practice to the limit of low collisionality and zero particle flux.

Transport simulations with the GLF23 model [37] and the ASTRA code [38] of the ITER standard scenario provide values of $R / L_{n}$ at mid-radius around 3 and a peaking factor of 1.45 [39]. GLF23 is found in rather good agreement with linear and nonlinear gyrokinetic results in the determination of the condition of zero particle flux as a function of plasma parameters in the collisionless limit [32]. Therefore, there is a rather large consensus in the theoretical predictions of the peaking of the density profile in the collisionless limit at zero particle flux. The theoretical prediction turns out to be very close to the value of density peaking obtained by the scaling of the density peaking in AUG and JET H-mode plasmas at ITER values, namely low collisionality and zero particle flux, as well as to the values of density peaking measured in low collisionality plasmas in AUG and JET. Such an agreement between theory and experimental observations is in our opinion a non-trivial result.

The investigation of the behaviour of passing and trapped electrons in the velocity space 
in nonlinear gyrokinetic simulations with the GENE code reveals that, in the case of ITG turbulence, the inward particle flux is produced by an inward pinch carried by trapped electrons. On the contrary, in the case of density gradient driven TEM turbulence, trapped electrons move outwards [32]. Since the inward pinch is carried by trapped electrons in ITG turbulence, a reduction of the pinch with increasing collisionality has to be expected. This reduction is actually found in both gyrofluid [5] and gyrokinetic [33, 40] calculations, which is in qualitative agreement with the experimental observation that density peaking decreases with increasing collisionality.

Transport simulations with the gyrofluid model GLF23 on a subset of AUG plasmas of the database are found to provide a collisionality dependence of density peaking which is in quantititative agreement with the experimental observations [5]. In that work, it is shown that collisionality is the crucial physics ingredient in the transport model to obtain an agreement with the experimental observations. On the contrary, quasi-linear and nonlinear flux-tube gyrokinetic simulations find that the inward pinch vanishes with increasing collisionality at collisionality values which are almost one order of magnitude lower than those achieved in the experiments [33, 40]. More recently, highly physics comprehensive global nonlinear gyrokinetic simulations find inward particle pinches also at experimental values of collisionality [41]. The latter result is certainly an important step towards a quantitative agreement between the results of nonlinear gyrokinetic simulations and the empirically determined collisionality dependence of density peaking. Such an agreement, however, is still missing at present.

\section{EXPERIMENTAL OBSERVATIONS ON IMPURITY TRANSPORT}

The increasing amount of experimental and theoretical evidence that the ITER density profile will not be flat, as presented in the previous sections, in combination with the need of using highly charged materials in the ITER plasma facing components and possibly a radiative mantel at the edge to mitigate heat loads on the walls, strongly motivates the study of impurity transport in the plasma core. This is undertaken intensively in both AUG and JET.

In AUG, the experimental study of impurity transport is receiving more and more importance since an increasing amount of plasma facing components is covered by tungsten- 
armour. Over an extended dataset of $\mathrm{H}$-mode discharges in AUG, it is observed that the brightness of the central bolometer line of sight is proportional to the brightness of a peripheral line of sight (tangent at $\rho \simeq 0.4$ ) in all plasmas which have central wave heating, ion cyclotron $(\mathrm{ICRH})$ or electron cyclotron $(\mathrm{ECH})$ resonance heating, or are sawtoothing, while plasmas without sawteeth and without central wave heating show a central brightness which is much larger [16]. These large differences can only be explained by strong central radiation of medium-Z to high-Z elements, which must have large density gradients inside $\rho \simeq 0.4$ in the latter case. These results motivated a set of dedicated experiments in AUG with Si laser ablation to quantify the effects [17]. The Si central diffusivity is strongly increased from neoclassical values up to almost one order of magnitude larger than neoclassical, when central ICRH or ECH heating is added. Correspondingly it is observed that the central effective heat conductivity increases by an order of magnitude, being at neoclassical levels before the switch-on of wave heating, and becoming anomalous afterwards. Fig. 3 shows an example of the results obtained in these experiments, comparing a case with NBI heating only, and cases with ECH on-axis and off-axis. The largest central diffusivities of Si are obtained in conditions of localized on-axis ECH. In these cases a very small pinch to diffusivity ratio is measured, in contrast with the case of NBI heating only, which shows a large inward pinch to diffusivity ratio, consistent with neoclassical theory [17]. From these observations, the use of central wave heating as a control tool to avoid tungsten accumulation has become regular on AUG, where the use of ECH is more efficient [16, 18, 21]. As revealed by the Si trace experiments [17], central wave heating not only can have the effect of reducing the central main ion density peaking, and therefore the neoclassical inward drift, but also of increasing the central diffusion above neoclassical levels. Moreover, the reduction of density peaking would not be sufficient to explain the suppression of impurity accumulation within neoclassical theory alone, as shown by specific neoclassical calculations with the NEOART code [42, 43].

Therefore, the general result in AUG is that if central heat transport is anomalous, high $Z$ impurities are not observed to accumulate. It must be emphasized that we refer to the very central region of the plasma, where the temperature gradients flatten, unless a strongly localized heat source is provided. In the confinement region, namely $0.3<r / a<$ 0.8 , impurity transport is regularly observed to be anomalous, and never accumulating. Moreover, in the presence of strong central electron heating, particle pinches very close to 
zero or even outward convection are observed.

The possibility of an inversion of the direction of the particle convection from inward to outward in conditions when the diffusivity is measured to be anomalous is interesting and has important applications in the case impurities have to be injected in the edge of the plasma to produce a radiative matel in order to reduce the heat loads on the walls, but their penetration in the bulk has to be avoided. The study of the direction of particle convection in response to ion or electron auxiliary heating has been studied recently at JET [22]. In H-mode plasmas, about $3 \mathrm{MW}$ of ICRH have been added to 12 to $14 \mathrm{MW}$ of NBI heating, applying two different heating schemes. In the first case, at small concentrations of $\mathrm{He}^{3}$, a minority heating $(\mathrm{MH})$ scheme is produced, delivering the largest amount of wave heating to the ions. In the second case, at larger concentrations of $\mathrm{He}^{3}$, a mode conversion (MC) scheme is obtained, delivering the largest amount of wave heating to the electrons. In response to the two different heating methods, a difference in the $\mathrm{Ni}$ trace behaviour is regularly observed. In the case of $\mathrm{MH}$, the diffusivity is close to the neoclassical levels in the very central part of the plasma column $(r / a<0.2)$, and strong accumulation, consistent with neoclassical predictions, is observed. In the confinement region, $0.3<r / a<0.8$, the diffusivity is at least one order of magnitude larger than neoclassical, and the same is true for the pinch velocity, directed inwards. However, the ratio $V / D$ remains around 1 , much smaller than in neoclassical theory (larger than 10). In contrast to the observations with $\mathrm{MH}$, in the case of MC diffusivity and pinch are observed above the neoclassical levels along the whole radius, and the convection term is measured to be smaller in absolute value than in the $\mathrm{MH}$ case and directed outwards. Observations of impurity convection directed outwards with auxiliary electron heating are also consistent with experimental results in TCV in the presence of strong ECH [44].

In JET, experiments are also performed in order to measure the diffusivity and pinch of impurities as a function of the impurity charge. A method which combines different diagnostics has been set which allows us to track simultaneously the dynamics of several different impurities [23]. In a set of $\mathrm{H}$-mode plasmas in JET, up to four impurities He, Ne, Ar and $\mathrm{Ni}$, are injected in the same discharge at a similar time [45]. In the confinement region, no strong dependence of either the diffusivity and the pinch to diffusivity ratio on the charge is observed. The diffusion coefficients are measured one order of magnitude larger than the neoclassical values, both in the core and at mid-radius, while the pinch to 
diffusivity ratio are measured much smaller than the neoclassical predictions. These results on the charge dependence of impurity transport provide a further experimental confirmation that, if the impurity transport is anomalous, no physics mechanism producing an impurity peaking which strongly increases with increasing charge is at play, unlike in neoclassical transport.

In summary, both AUG and JET experimental results on impurity transport provide a general consistent picture of the behaviour of the impurities in tokamak plasmas. In the very central region of the plasma column, impurity transport can be both neoclassical or anomalous. In the confinement region, impurity transport is always observed to be anomalous. If the transport is anomalous, namely in the presence of an impurity diffusivity above the neoclassical prediction, the impurity profiles never show strong accumulation, and have a peaking which is comparable to that of the electron density profile. This behaviour is observed to be rather independent of the charge of the impurity, with consequently rather flat concentration profiles for all charges. Moreover, in the presence of an anomalous impurity diffusivity, evidence of the existence of an anomalous pinch is observed. Interestingly, the anomalous convection can be directed both inwards or outwards, and there is an agreement among the experiments indicating that conditions of electron heating produce smaller inward pinches with respect to ion heating, or even small outward convection, with consequent hollow impurity density profiles. While the present observations are consistent, we acknowledge that they are still rather fragmentary. Additional systematic experimental efforts are required to better identify not only domains of parameters at which anomalous impurity transport, and therefore no accumulation, is expected, but also specific parameter dependences of both diffusivity and pinch.

\section{IMPURITY TRANSPORT PRODUCED BY CORE MICROINSTABILITIES}

Core impurity transport can have important consequences on the plasma performance, up to a radiative collapse. However, the effort of going beyond the neoclassical theory and provide a theoretical description of the experimental observations also when they exceed the neoclassical predictions started only very recently.

The experimental results presented in the previous section allow the identification of two general requirements that a theoretical prediction of anomalous impurity transport must 
satisfy in order to not disagree with the experimental observations. Firstly, it should not involve mechanisms of impurity pinch which increase with increasing charge more strongly than the diffusivity, leading to strong accumulation. Secondly, it must be such to provide a convection directed both inwards and outwards depending on the plasma parameters. Here, we show that these requirements are fulfilled by the transport produced by microinstabilities like ITG and TEM, which are recognized as the main cause of anomalous heat transport in tokamak plasmas.

In a mainly electrostatic turbulent plasma, three dominant mechanisms transporting impurities can be indentified. The first is the $E \times B$ advection and compression. The advection contributes to the diagonal transport, while the compression provides a charge independent inward pinch often called curvature or q-pinch [46, 47], which corresponds to the same pinch mechanism identified by the Turbulent Equipartition theory [48, 49]. This pinch mechanism has also been indentified recently in the framework of the theory of ratchet processes [50].

The second mechanism, usually called thermodiffusion, comes from the coupling with temperature fluctuations via the $\nabla B$ and curvature drift and causes a pinch term which is proportional to the logarithmic impurity temperature gradient. It decreases with increasing impurity charge as a consequence of the inverse charge dependence of the curvature drift [51-54]. This mechanism has the property of reversing its direction as a function of the direction of propagation of the turbulence. It is directed inwards for modes propagating in the electron diamagnetic direction, whereas it is directed outwards from modes propagating in the ion diamagnetic direction. Hence, it is in opposite directions with respect to the equivalent mechanism transporting electrons [7, 46, 47].

The third mechanism is connected with the parallel compression of parallel velocity fluctuations produced along the field line by the fluctuating electrostatic potential [53]. This convective term is proportional to the ratio of the charge to the mass number of the impurity, and therefore does not vanish for sufficiently ionized heavy impurities. This mechanism is directed inwards for instabilities rotating in the ion diamagnetic direction, while it is directed outwards for instability directed in the electron diamagnetic direction.

The total turbulent pinch results as a complex combination of at least these three mechanisms and therefore is connected with the dominant instabilities in the plasma and finally with its turbulent state. We observe that the transport produced by these mechanisms ful- 
fils both the requirements derived from the analysis of the experimental results, namely no strong accumulation increasing with increasing charge is predicted, and convective mechanisms directed both inwards and outwards are possible.

In Fig. 4, the effects of the different mechanisms are shown on an experimental case. The $\mathrm{Z}$ dependence of the pinch to diffusivity ratio $-R V / D$ of a trace impurity is computed with GS2 for typical plasma parameters in the radial interval $0.35<r / a<0.55$ of a high density $\left(n_{e}=910^{1} 9 \mathrm{~m}^{-3}\right)$ AUG H-mode plasma with central ECH. The calculation of $V$ and $D$ for a trace impurity is obtained following the method described in [53]. Circles show the dependence obtained when the actual plasma parameters are used, namely $\epsilon=0.16, q=1.4$, $s=0.8, R / L_{T e}=6.5, R / L_{T i}=5.0, R / L_{n}=2.0, T_{e}=T_{i}$, where the impurity temperature profile has been taken equal to the deuterium temperature. The dominant instability is found to be an ITG, despite $R / L_{T e}>R / L_{T i}$, due to the relatively high collisionality and the rather small density gradient of this plasma. The curve with triangles shows the results of similar calculations but considering a flat temperature gradient for the trace impurity. This allows us to single out the contribution due to thermodiffusion (curve with crosses in the figure), which is found to be directed outwards, as the negative sign indicates, consistent with the ion diamagnetic direction of rotation of these modes. Moreover the curve with squares shows the results obtained in the case that a dominant TEM instability is present, which has been obtained by increasing the logarithmic electron temperature gradient up to 9 , and decreasing the collisionality by a factor of 5 . In this case the thermodiffusion contribution (not shown) is directed inwards, and for large values of $\mathrm{Z}$, where thermodiffusion becomes small, the outward parallel compression term balances the inward $E \times B$ compression pinch to provide a total pinch very close to zero and a flat or even slightly hollow density profile for highly charged impurities. The effect of the parallel compression is also visible for $\mathrm{W}$ when an experimentally observed ionisation stage, namely $W^{46+}$, is considered, as shown in the figure by the diamond point $(Z=46$ and $A=184)$, for the experimental input parameters. The reduction of the pinch to diffusion ratio with respect to the curve obtained assuming $A=2 Z$ is due exclusively to a reduction of the inward pinch and is attributed to the reduction of the pinch due to parallel compression, which is directed inwards for ITG instabilities. The diffusion coefficient is instead the same as the one obtained in the case $A=2 Z$, showing that the diffusion coefficient becomes independent of $A$ at large values of $A$. The pinch is caused by the reduction of the $Z / A$ ratio to which the parallel compression pinch is 
proportional. In general, the reduction of the pinch to diffusion ratio of highly charged trace impurities predicted in the case of dominant TEM instabilities is in qualitative agreement with the observation of an efficient flattening of the impurity concentration profiles in the presence of auxiliary electron heating observed in tokamaks [17, 22, 44]. The overall result that turbulent transport does not predict mechanisms of impurity pinching which strongly increase with increasing $\mathrm{Z}$, as instead it is predicted by neoclassical theory, is in agreement with the whole set of experimental observations in AUG and JET.

\section{CONCLUSIONS}

The comparison and combination of experimental observations from different devices, such as AUG and JET, on particle and impurity transport allows a more robust identification of general plasma behaviours and of their parametric dependences.

By combining observations of AUG and JET H-mode plasmas, a database has been built for the statistical study of parameters describing density peaking in the two devices. Collisionality is found to be the statistically most relevant parameter in multivariable regressions for the density peaking. The contribution of the beam fuelling is never negligible, but alone is found to be unable to describe the full observed variation of density peaking. These results, in combination with the observation of peaked density profiles with wave heating only at low collisionality, can only be explained by the existence of an anomalous inward pinch in low collisionality $\mathrm{H}$-mode plasmas. A scaling for density peaking which include collisionality in the regression variables predict a peaked density profile for ITER, with a peaking factor $n_{e}\left(\rho_{p o l}=0.2\right) /\left\langle n_{e}\right\rangle=1.45$. Such a peaking factor corresponds to a logarithmic density gradient at mid-radius $R / L_{n}$ between 2.5 and 3.5, which is close to the $R / L_{n}$ value fulfilling the condition of zero particle flux as computed by gyrokinetic and gyrofluid codes in the collisionless limit at experimentally observed values of the logarithmic temperature gradients. For smaller values of $R / L_{n}$, a total particle flux directed inwards is obtained in the theoretical calculations, carried by trapped electrons. This pinch is found to decrease with increasing collisionality in qualitative agreement with the experimental observations. However a quantitative agreement between the experimentally observed density peaking dependence on collisionality and nonlinear gyrokinetic simulations is still missing.

Both the experimental and theoretical results indicate that the density profile in the 
ITER standard scenario will not be flat, in contrast to usual assumptions [55]. A peaked density profile has positive consequences on the fusion power, which is found to increase by approximately $30 \%$ at the predicted values of density peaking [12, 39] with respect to a flat density profile, keeping fixed the temperature profiles and impurity concentration profiles.

A peaked density profile raises concerns about the behaviour of heavy impurities. AUG and JET experimental results on impurity transport show that if the transport is anomalous, no strong accumulation of highly charged impurities takes place, in contrast to the behaviour predicted by neoclassical theory. This experimental result agrees with the theoretical finding that no pinch mechanism producing strong accumulation is predicted by core microinstabilities like ITG modes and TEMs. These results are also promising for a burning plasma, provided the central $\alpha$ heating is large enough to drive a turbulent transport up to the innermost region of the plasma column. Finally, anomalous impurity convection is observed in both inward and outward directions. In particular, small or even outward convection is observed in conditions of central electron heating, in qualitative agreement with the theoretical predictions of an outward impurity convection in the case of strong electron temperature gradient driven TEM microinstabilities.

\section{Acknowldgements}

The Authors are grateful to W. Dorland and M. Kotschenreuther for providing the gyrokinetic code GS2, and to R.E. Waltz, J. Kinsey and G.M. Staebler for the transport model GLF23. 
[1] O.Gruber, H.-S. Bosch, S. Günter et al., Nucl Fusion 39, 1321 (1999).

[2] J. Pamela, E.R. Solano, and JET EFDA Contributors, Nucl. Fusion 43, 1540 (2003).

[3] J. Stober, R. Dux, O. Gruber et al., Nucl. Fusion 43, 1265 (2003).

[4] C. Angioni, A.G. Peeters, G.V. Pereverzev, F. Ryter, G. Tardini, and ASDEX Upgrade Team, Phys. Rev. Letters 90, 205003 (2003).

[5] C. Angioni, A.G. Peeters, G.V. Pereverzev, F. Ryter, G. Tardini, and ASDEX Upgrade Team, Phys. Plasmas 10, 3225 (2003).

[6] L. Garzotti, X. Garbet, P. Mantica et al., Nucl. Fusion 43, 1829 (2003).

[7] C. Angioni, A.G. Peeters, X. Garbet, A. Manini, F. Ryter and ASDEX Upgrade Team, Nucl. Fusion 44, 827 (2004).

[8] M. Valovič, R. Budny, L. Garzotti et al., Plasma Phys. Control. Fusion 46, 1877 (2004).

[9] H. Weisen, A. Zabolotsky, C. Angioni et al., Nucl. Fusion 45, L1 (2005).

[10] C. Angioni, A.G. Peeters, F. Ryter, F. Jenko, G.D. Conway, T. Dannert, U.H. Fahrbach, M. Reich, W. Suttrop, L. Fattorini, Phys. Plasmas 12, 040701 (2005).

[11] A. Zabolotsky, H. Weisen, A. Karpushov, Nucl. Fusion 46, 594 (2006).

[12] H. Weisen, A. Zabolotsky, M. Maslov, M. Beurskens, C. Giroud, D. Mazon, Plasma Phys. Control. Fusion 47, A457 (2006).

[13] L. Garzotti, M. Valovič, X. Garbet, P. Mantica, V. Parail, Nucl. Fusion 46, 994 (2006).

[14] C. Angioni, H. Weisen, O.J.W.F. Kardaun et al., Scaling of density peaking in H-mode plasmas based on a combined database of AUG and JET observations, submitted to Nucl. Fusion (2006).

[15] M. Valovič, L. Garzotti, I. Voitsekhovitch, M. Beurskens, X. Garbet, C. Giroud, D. Keeling, On the correlation between density profile and particle flux in $H$-mode tokamak plasma and implication for ITER, submitted to Nucl. Fusion (2006).

[16] R. Neu, R. Dux, A. Geier, H. Greuner, K. Krieger, H. Maier, R. Pugno, V. Rohde, S.W. Yoon, Journ. Nucl. Materials 313, 116 (2003).

[17] R. Dux, R. Neu, A.G. Peeters, G. Pereverzev, A. Mück, F. Ryter, J. Stober, Plasma Phys. Control. Fusion 45, 1815 (2003).

[18] R. Dux, Fusion Science and Technology 44, 708 (2003).

[19] M.E. Puiatti, M. Valisa, M. Mattioli et al., Plasma Phys. Control. Fusion 45, 2011 (2003). 
[20] R. Dux, C. Giroud, K.-D. Zastrow, Nucl. Fusion 44, 260 (2004).

[21] R. Neu, R. Dux, A. Kallenbach, et al., Nucl. Fusion 45, 209 (2005).

[22] M.E. Puiatti, M. Valisa, C. Angioni, L. Garzotti, P. Mantica, M. Mattioli, L. Carraro, I. Coffey, C. Sozzi, Phys. Plasmas 13, 042501 (2006).

[23] C. Giroud, R. Barnsley,P. Buratti et al., Experimental determination of $Z$ dependence of impurity transport on JET, submitted to Nucl. Fusion (2006).

[24] R. Aymar V.A. Chuyanov, M. Huguet et al., Nucl. Fusion 41, 1301 (2001).

[25] S.P. Hirshmann, D.J. Sigmar, Nucl. Fusion 21, 1079 (1981).

[26] D. Bailey, Report JET-R (1998) 04;

P.M. Stubberfield and M.L. Watkins Report JET-DPA(06)/87 (1987);

M. Cox Culham Report for JET KR5-33-04 (1984).

[27] G.G. Lister, "FAFNER - A Fully 3-D Neutral Beam Injection Code Using Monte Carlo Methods” IPP-Report 4-222, Garching, Germany (1985).

[28] O.J.W.F. Kardaun, Classical Methods of Statistics (Springer Verlag, Berlin Heidelberg, 2005) p. 113.

[29] M. Greenwald, D. Ernst, A. Hubbard, J.W. Hughes, Y. Lin, J. Terry, S. Wukitch, K. Zhurovich, Bull. Am. Phys. Soc. Vol. 51, no 7, 139 (2006), 48th A.P.S. - D.P.P. Meeting 2006, JO1 3.

[30] M. Kotschenreuther, G. Rewoldt, and W.M. Tang, Comput. Phys. Commun. 88, 128 (1995).

[31] W. Dorland, F. Jenko, M. Kotschenreuther, B.N. Rogers, Phys. Rev. Lett. 85, 5579 (2000).

[32] F. Jenko, T. Dannert, and C. Angioni, Plasma Phys. Control. Fusion 47, B195 (2005).

[33] C. Angioni, A.G. Peeters, F. Jenko, T. Dannert, Phys. Plasmas 12, 112310 (2005).

[34] D.R. Ernst, P.T. Bonoli, P.J. Catto et al., Phys. Plasmas 11, 2637 (2004).

[35] A.G. Peeters, C. Angioni, M. Apostoliceanu, F. Jenko, F. Ryter, Phys. Plasmas 12, 022505 (2005).

[36] A.M. Dimits, G. Bateman, M.A. Beer et al., Phys. Plasmas 7, 969 (2000).

[37] R.E. Waltz, G.M. Staebler, W. Dorland, G.W. Hammett, M. Kotschenreuther,and J.A. Konings, Phys. Plasmas 4, 2482 (1997).

[38] G.V. Pereverzev, P.N. Yushmanov, "ASTRA Automated System for TRansport Analysis in a Tokamak", Report IPP 5/98, Max-Planck-Institut für Plasmaphysik, Garching, 2002.

[39] G.V. Pereverzev, C. Angioni, A.G. Peeters, O.V. Zolotukhin, Nucl. Fusion 45, 221 (2005). 
[40] C. Estrada-Mila, J. Candy, R.E. Waltz, Phys. Plasmas 12, 022305 (2005); Erratum Phys. Plasmas 12, 049902 (2005).

[41] C. Estrada-Mila, J. Candy, R.E. Waltz, Phys. Plasmas 13, 074505 (2006);

[42] A.G. Peeters, Phys. Plasmas 7, 268 (2000).

[43] R. Dux, A.G. Peeters, Nucl. Fusion 40, 1721 (2000).

[44] E. Scavino, J. Bakos, H. Weisen, Plasma Phys. Control. Fusion 46, 857 (2004).

[45] C. Giroud, Proceedings of the 21st IAEA Conference on Fusion Energy, Chengdu, China, 2006, edited by M. Spak (International Atomic Energy Agency, Vienna, 2006), EX/8-3.

[46] J. Weiland, A.B. Jarmen, H. Nordman, Nucl. Fusion 29, 1810 (1989).

[47] X. Garbet, L. Garzotti, P. Mantica, H. Nordman, M. Valovič, H. Weisen, C. Angioni and JET EFDA Contributors, Phys. Rev. Lett. 91, 035001 (2003).

[48] M.B. Isichenko,A.V. Gruzinov, P.H. Diamond, Phys. Rev. Lett. 74, 4436 (1995).

[49] D.R. Baker and M. Rosenbluth, Phys. Plasmas 5, 2936 (1998).

[50] M. Vlad, F. Spineanu, S. Benkadda, Phys. Rev. Lett. 96, 085001 (2006).

[51] M. Frojdh, Liljestrom, H. Nordman, Nucl. Fusion 32, 419 (1992).

[52] X. Garbet, N. Dubuit, E. Asp, Y. Sarazin, C. Bourdelle, P. Ghendrih, G.T. Hoang, Phys. Plasmas 12, 082511 (2005).

[53] C. Angioni and A.G. Peeters, Phys. Rev. Lett. 96, 095003 (2006).

[54] N. Dubuit, private communication (2006) (http://www-fusionmagnetique.cea.fr/ttf2006/prog/drafts/049.pdf).

[55] V.S. Mukhovatov, Y. Shimomura, A. Polevoi, Nucl. Fusion 43, 942 (2003). 
Table 1. Statistical relevance of plasma parameters in regressions for the density peaking in which collisionality, Greenwald fraction and major radius are alternatively included or excluded in the regression variables.

\begin{tabular}{|l||l|l|l|c|c|c|c|c|c|c|}
\hline \hline no $F_{G R}$ & $\Gamma_{N B I}^{*}$ & $\ln \nu_{e f f}$ & $F_{G r}$ & $\rho_{*}$ & $\beta$ & $q_{95}$ & $\delta$ & $T_{e 2} /\left\langle T_{e}\right\rangle$ & $R_{\text {geo }}$ & RMSE \\
no $F_{G R} \& R_{\text {geo }}$ & 0.077 & -0.098 & & 0.027 & -0.048 & -0.013 & -0.016 & 0.001 & 0.049 & 0.113 \\
no $\nu_{\text {eff }}$ & 0.096 & -0.127 & & -0.003 & -0.032 & -0.018 & -0.003 & -0.006 & & 0.114 \\
no $\nu_{\text {eff }} \& R_{\text {geo }}$ & 0.083 & & -0.058 & 0.028 & -0.035 & 0.003 & -0.017 & -0.012 & 0.094 & 0.122 \\
All vars & 0.077 & -0.115 & 0.028 & 0.042 & -0.063 & -0.015 & -0.025 & 0.002 & 0.057 & 0.113 \\
no $R_{\text {geo }}$ & 0.066 & -0.136 & 0.012 & 0.001 & -0.037 & -0.019 & -0.006 & -0.006 & & 0.114 \\
\hline \hline
\end{tabular}

Table 1. 
FIG. 1: (Color online) Density peaking as a function of collisionality (a), Greenwald fraction (b) , $\rho_{*}(\mathrm{c})$, and beam source parameter $\Gamma_{N B I}^{*}(\mathrm{~d})$, from the combined database of AUG and JET $\mathrm{H}$-mode plasmas.

FIG. 2: (Color online) Particle flux as a function of the normalised logarithmic density gradient $R / L_{n}$ for different values of the normalised logarithmic temperature gradient $R / L_{T}=R / L_{T i}=$ $R / L_{T e}$, computed with the linear version of the GS2 code [26] and the quasi-linear model in [29] and the nonlinear GENE code [28] for a single value of the logarithmic temperature gradient (a); corresponding values of $R / L_{n}$ at the null of the particle flux as a function of $R / L_{T}$, with highlighted regions of different dominant instabilities, and in dark grey the window of logarithmic temperature gradients regularly measured in AUG and JET H-mode plasmas at mid-radius (b).

FIG. 3: (Color online) Diffusion coefficients (a), pinch velocities (b) of a Si trace and power balance conductivities, together with the neoclassical predictions (c), for a set of $0.8 \mathrm{MA} \mathrm{H}-$ mode AUG plasmas, with 5 MW NBI heating only and with additional ECH [16]. 
FIG. 4: (Color online) Normalized pinch to diffusion ratio as computed by a quasi-linear model with the GS2 code, as a function of $Z$, with $A=2 Z$ for different choices of input parameters as explained in the text and related to the experimental profiles of an AUG H-mode plasma with central ECH. 

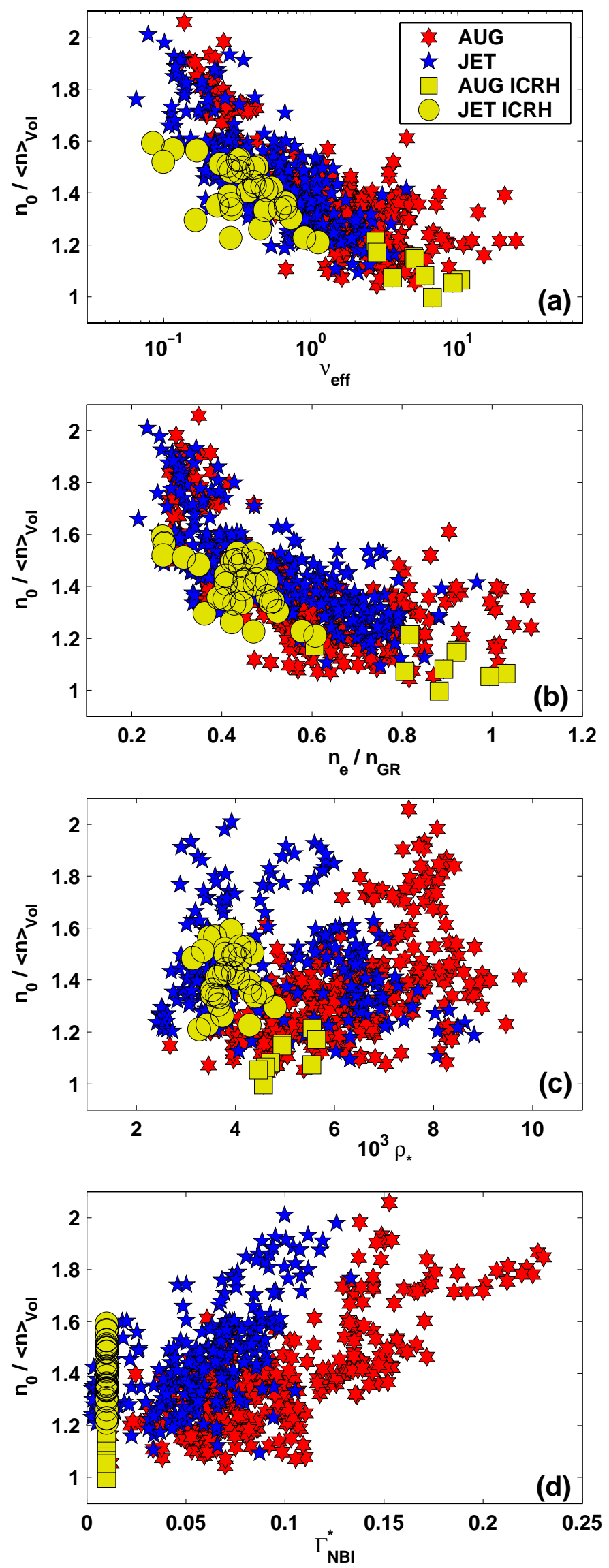
Figure 1
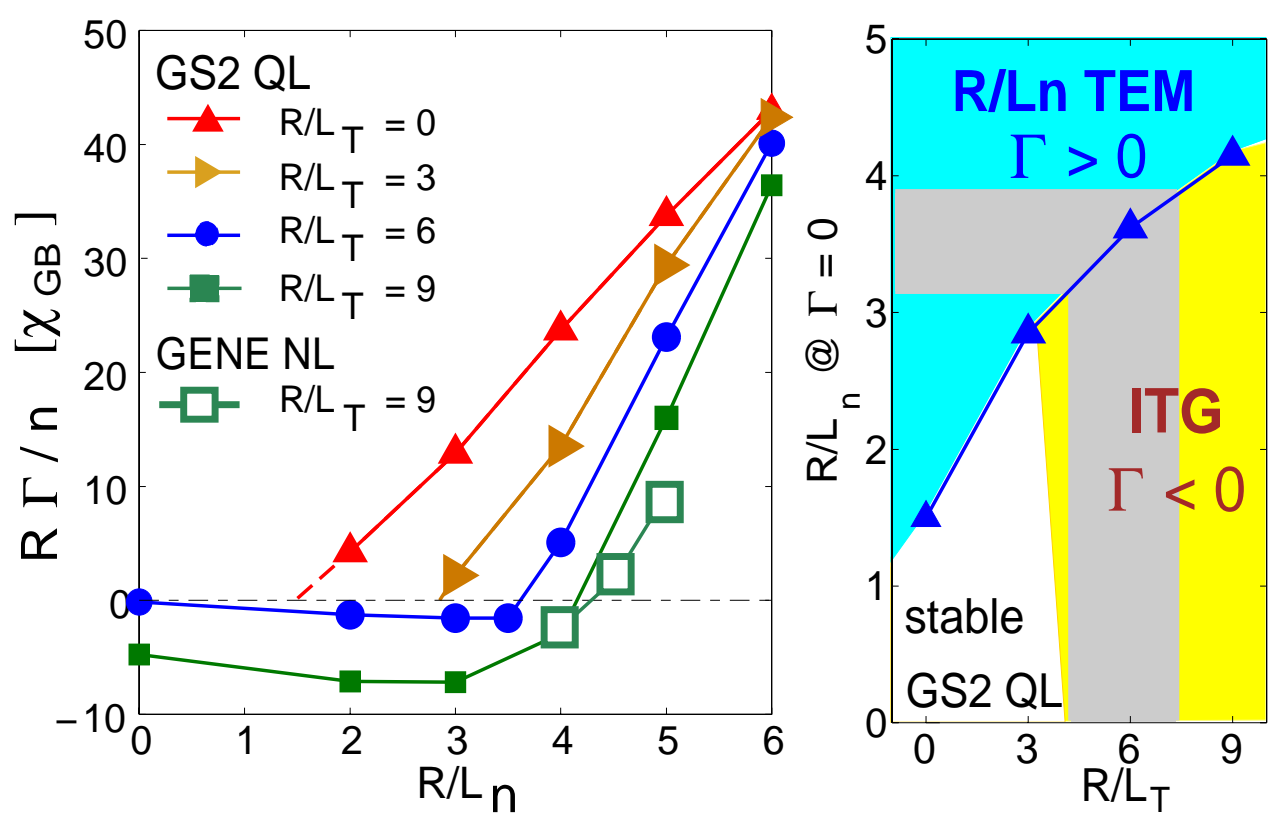

Figure 2 


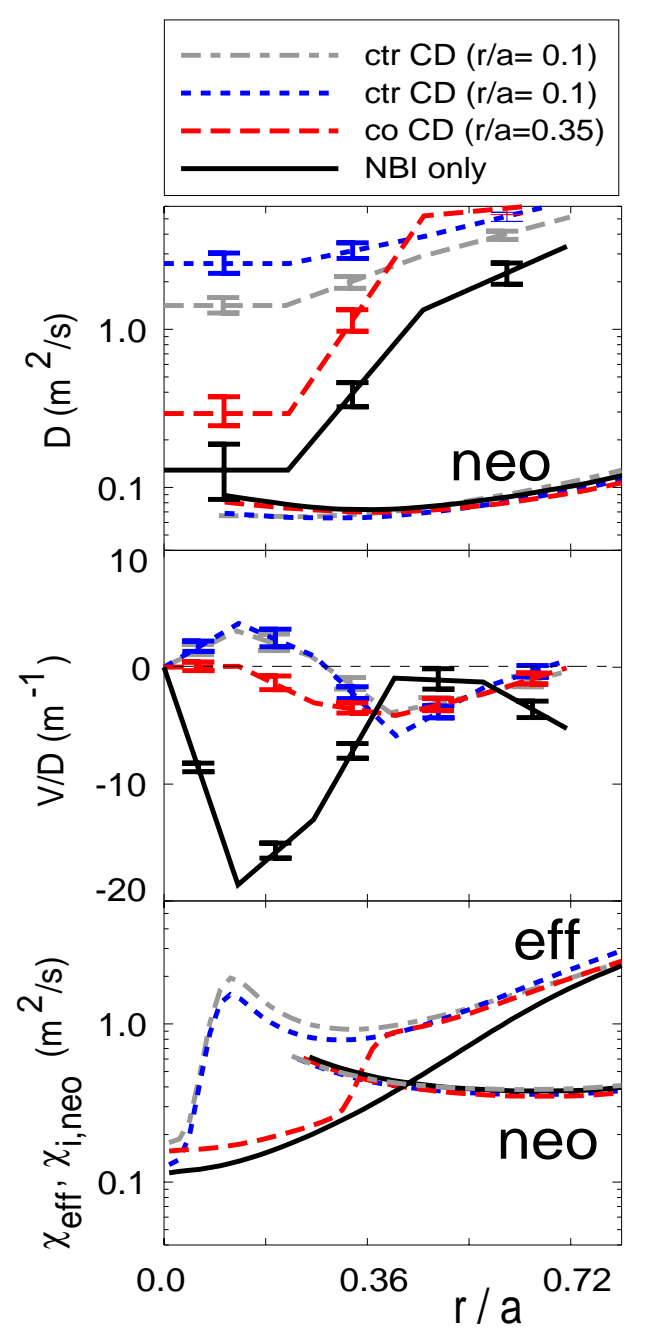

Figure 3

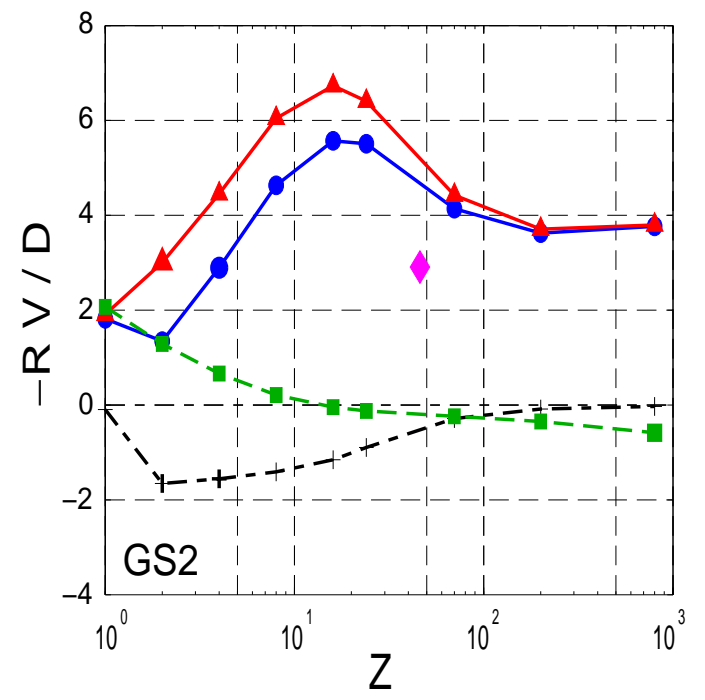

Figure 4 